\title{
TRIMS AND ANTIVIRAL IMMUNITY IN FISH: A FINTRIM INDUCING CONSTITUTIVE IFN SIGNALING IS EXPRESSED AT SURFACES EXPOSED TO PATHOGENS
}

\author{
Christelle Langevin $^{\S 1}$, Elina Aleksejeva ${ }^{1}$, Armel Houel ${ }^{1}$, Corinne Torhy ${ }^{1}$, Jean-Pierre \\ Levraud $^{2,3}$, Pierre Boudinot ${ }^{\$ 1}$
}

${ }^{1}$ INRA, Université Paris Saclay, Virologie et Immunologie Moléculaires, 78352 Jouy-en-Josas, France

${ }^{2}$ Institut Pasteur, Unité Macrophages et Développement de l'Immunité, rue du Docteur Roux, Paris, France

${ }^{3}$ CNRS, URA 2578 Paris, France

\begin{abstract}
The tripartite-motif-protein (TRIM) family includes many key components of the antiviral arsenal expressed in mammals; antiviral TRIMs mediate intrinsic viral restriction at diverse points of the viral cycle, or positively regulate innate immune signaling pathways. The TRIM family is ancient and has been greatly diversified in vertebrates and especially in fish. Our previous survey of fish TRIM genes in fish identified subsets with different evolutionary dynamics, with several subsets highly diversified like the finTRIMs. We show here that a zebrafish finTRIM gene constitutively expressed in the gills, skin and pharynx, encodes a protein that strongly up-regulates the type I interferon (IFN) pathway. While it is not IFN-inducible, its in vivo expression in gills of healthy fish correlates with that of type I IFN. In vitro, overexpression of this finTRIM induces IFN and IFN-stimulated gene expression, and affords protection against different enveloped and non-enveloped RNA viruses. This antiviral activity is IFN-dependent, and is abolished by a dominant negative IRF3 mutant. Our work indicates that TRIM proteins contribute to the establishment of antiviral immunity, possibly by permanent type I IFN stimulation in exposed tissues. Hence TRIMs might create a local anti-viral environment at sites exposed to pathogens, a mechanism participating to the regionalization of immunity. Our data also reveal that TRIMs were involved in antiviral immunity before the divergence between bony fish and tetrapods, early in Vertebrate evolution.
\end{abstract}

\section{KEYWORDS}

TRIM; antiviral innate immunity; interferon; regionalization of immune defense

$\S$ Corresponding authors :

CL: tel +33134652589 email christelle.langevin@jouy.inra.fr

or PB: tel +33134652585 email pierre.boudinot@jouy.inra.fr 